\title{
the Benefits of Additional Defibrillator in Heart Failure Patients with Cardiac Resynchronization Therapy: A Meta-analysis of Observational Studies
}

\author{
Yuxiang Long ${ }^{1}$, Diyu Cui ${ }^{1}$, and Zengzhang Liu ${ }^{1}$ \\ ${ }^{1}$ Chongqing Medical University Affiliated Second Hospital
}

June 2, 2020

\begin{abstract}
Abstract Background: Current guidelines did not provide recommendations on indications of an additional implantable cardioverterdefibrillator (ICD) to patients receiving cardiac resynchronization therapy (CRT), and it still remains controversial due to lack of evidence from randomized controlled trials (RCTs). Method: PubMed, Embase and Cochrane CENTRAL from the inception to May 2020 were systematically screened for studies reporting on the comparison of CRT-defibrillator (CRT-D) and CRT-pacemaker (CRT-P), focusing on the adjusted hazard ratio (aHR) of all-cause mortality. We pooled the effects using a random-effect model. Results: Twenty-one studies encompassing 69919 patients were included in this meta-analysis. With no restriction to characteristics of including population, CRT-D was associated with a lower all-cause mortality compared with CRT-P significantly (aHR: 0.79, 95\%CI: 0.72-0.88, I2=40.5\%, P<0.0001). This mortality benefit was also observed in patients with ischemic cardiomyopathy (ICM) (HR: 0.74, 95\%CI: 0.64-0.86, I2=0\%, $\mathrm{P}$ 0.0001). However, there is no significant difference in patients with non-ischemic cardiomyopathy (NICM) (HR: 0.91, 95\%CI: 0.82-1.01, I2=0\%, P=0.087), older age (age [?]75 years, aHR: 0.96, 95\%CI: $0.83-1.12, \mathrm{I} 2=0 \%, \mathrm{P}=0.610$ ). Subgroup analysis was performed and indicated that there is no mortality benefit of CRT-D for primary prevention (aHR: 0.92, 95\%CI: 0.74-1.14, I2=0\%, P=0.444). Conclusion: After adjusted the differences in clinical characteristics, additional ICD therapy was associated with a reduced all-cause mortality in patients receiving CRT, especially in patients with ICM. However, our work suggested that additional ICD may not be applied to elderly, NICM patients or for primary prevention. Keyword: heart failure; cardiac resynchronization therapy; defibrillator; all-cause mortality; meta-analysis
\end{abstract}

\section{Introduction}

Heart failure (HF), a major public health issue, is becoming more prevalent due to the aging and longer survival of cardiovascular patients [1]. As HF progressing, pump failure and life-threatening ventricular arrhythmias are major causes of death [2]. Implantable cardioverter defibrillator (ICD) and cardiac resynchronization therapy (CRT) are widely applied to mild or severe HF patients with reduced ejection fraction and a wide QRS duration to reduce all-cause mortality [3].

The COMPANION trial has demonstrated that CRT with or without ICD (CRT-D or CRT-P) can decrease the risk of all-cause mortality and CRT-D was associated with a lower mortality, but there is no definitive comparison between CRT-D and CRT-P [4]. Then, plenty of observational studies reported the various outcome of CRT-D comparing to CRT-P, and a meta-analysis including nineteen studies presented that the addition of an ICD is associated with a reduction in the risk of all-cause mortality [5]. However, we cannot deny significant differences in clinical characteristics between two groups in these studies. Patients in CRT-P group were generally older age, more comorbidities and more frailty, which may introduce selection bias and resulting the consequence to be incorrect [6]. 
Due to lack of strong randomized evidence, it still remains controversial whether CRT-D can bring benefits compared with CRT-P. Current guideline of the European Society of Cardiology recommend that the clinician should choose CRT-P or CRT-D whichever they consider appropriate [7]. However, this choice in practice may put clinicians in a dilemma. So far, several studies focusing on the comparison of CRT-D and CRT-P in all-cause mortality has been reported, which used Cox multivariate analysis or propensity score matching to minimize the influence of clinical differences between groups[6,8-19]. Therefore, we aim to review those studies to clarify the association of all-cause mortality and additional ICD in patients receiving CRT, and optimize the management of patients based on characteristics.

\section{Method}

\section{Search strategy}

This meta-analysis was conducted in compliance with the Preferred Reporting Items for Systematic reviews and Meta-analysis (PRISMA), and we have registered this work online at its inception on PROSPERO to minimize duplicate efforts.

We searched the Embase, PubMed and Cochrane CENTRAL from the inception dates to May 2020, using the key words: ((heart failure) or (Cardiac Failure) or (Heart Decompensation)) and ((Cardiac resynchronization therapy) or (CRT)) AND ((Defibrillator) OR (Defibrillation) OR (CRT-D))). There was no language restrictions and the literatures were searched by one researcher. We also checked the bibliographies of potentially relevant studies and reviews for additional studies.

\section{Study selection}

Two independent reviewers (Y.L and D.C) screened the citations through the title and abstract, and we make mutual agreement procedural to reach a consensus if divergences exist. Studies met the following explicit selection criteria as follow were evaluated as complete reports: 1) English language publication; 2) randomized controlled trials or observational trials; 3) the subjects of interest was heart failure patients undergoing CRT with or without defibrillator; 4) the outcome was all-cause mortality; 5) a hazard ratio (HR) adjusted by Cox multivariate analysis was available.

The standard of exclusion criteria includes: case reports, case series, editorials, literature review, conference abstract, non-English articles and duplicate reports (we selected the study reporting the largest sample of patients in these cases).

\section{Data extraction and quality assessment}

Two independent reviewers (Y.L and D.C) extracted the data on prespecified forms: authors, year of publication, study design, sample size, research center, follow-up time, time of CRT implantation, indication, adjusted covariates, endpoints and aHR value with its $95 \%$ confidence interval (CI). When different adjusted models existed in one study, we extracted the HR value with the greatest number of adjusted confounding variables. In instances when incomplete data were obtained, the authors were contacted in writing for permission to obtain further data.

The quality of each study was assessed independently by the same two investigators using the NewcastleOttawa scale (NOS), a nine-star scale for nonrandomized study quality assessment in meta-analyses. Briefly, this scale has three broad perspectives as follows: selection, comparability and outcome of studies. Studies awarded more than six stars were considered high-quality studies.

\section{Statistical analysis}

Continuous variables are presented using mean and standard deviation or median and interquartile range. Categorical data are presented as numbers and percentages. Random-effect meta-analysis was performed because of the observational design of the included studies. HRs and 95\%CI adjusted by Cox multivariate analysis was considered the effect size, which evaluate the risk of all-cause mortality between CRT-D and CRT-P. Heterogeneity among studies was assessed by the Cochran's Q test and was considered significant 
when $\mathrm{P}<0.10$. The $\mathrm{I}^{2}$ statistic was used to evaluate the proportion of variance caused by heterogeneity in observed variance. Subgroup analyses were performed in accordance to the differences of follow-up duration, indication of CRT-D, region of research center and year of publication between studies. Sensitivity analysis was performed by removing one study at a time. Publication bias was estimated using funnel plots and the regression test of Egger or Begg. Statistical analyses were all performed with STATA 14 (Stata Corp LP, College Station, Texas).

\section{Result}

\section{Description of included studies}

The flow diagram of study selection is shown in Figure 1. Through an electronic search of three databases (PUBMED, EMBASE and CENTRAL) by keywords, 4317 citations were screened after 505 duplicates removed. Full-text review was performed in 47 studies, of which 26 studies did not meet the inclusion criteria and leaving 21 studies in the final qualitative and quantitative analysis.

The baseline characteristic of individual studies and patients are presented in Table 1 and Supplementary Table 1, respectively. In consideration of different studies from the same research team or database reporting various endpoints, we selected one with largest sample size and calculated that a total of 69919 patients were included in this meta-analysis, and the mean follow-up duration varied from 1.8 to 5 years. All studies included were observational design, in which 5 studies were prospective and the others were retrospective. The majority of included studies are from centers in European and the USA, and all patients have received CRT-P or CRT-D between 1999-2017. Trials were quite variable in association with sample size. The smallest of the studies included only 135 subjects, and the largest trial enrolled 50084 subjects.

Of those included studies, 17 of them reported adjusted HR of all-cause mortality [6,8-10,12-14,16,17,19-27], and 4 studies reported HRs after propensity score matching $[8,14,17,19]$. Adjusted covariates of each studies are listed in Supplementary Table 2. CRT-D implantation as a primary prevention therapy for suddencardiac death was clearly stated in 5 studies $[6,12,14,19,25]$. We assessed the quality of studies by using NOS, and only four studies had a score of [?]7 (Supplementary Table 3). Meanwhile, we pooled adjusted HR of all-cause mortality in patients with NICM, ICM and older age ([?]75 years) to explore the optimal selection of individual device.

\section{Primary outcome: all-cause mortality in all patients}

The pooled aHR of studies suggested a lower all-cause mortality risk in patients receiving CRT-D compared with those receiving CRT-P (HR: 0.79, 95\%CI: 0.72-0.88, $\mathrm{I}^{2}=40.5 \%, \mathrm{P}<0.0001$ ) (Figure 2). Funnel plots for primary outcome was a little bit of asymmetric but both Egger's test and Begg's test did not indicate a significant bias $(\mathrm{P}=0.084)$. However, repeated analysis using $\mathrm{HR}$ of propensity matching cohort revealed that there was no significant difference in all-cause mortality between CRT-D and CRT-P (HR: 0.90, 95\%CI: $0.68-1.18, \mathrm{I}^{2}=43.4 \%, \mathrm{P}=0.438$ ) (Figure 3 ). Further, we conducted a sensitivity analysis to demonstrate the robustness of our conclusion by removing one study at a time.

\section{Secondary outcome: all-cause mortality in specific population}

In patients with ICM, CRT-D was associated with a lower all-cause mortality risk compared with CRTP (HR: 0.74, 95\%CI: 0.64-0.86, $\mathrm{I}^{2}=0 \%, \mathrm{P}_{j} 0.0001$ ) (Figure 4A). However, this benefit of CRT-D was not confirmed in patients with non-ischemic cardiomyopathy (HR: $0.91,95 \%$ CI: $0.82-1.01, \mathrm{I}^{2}=0 \%, \mathrm{P}=0.087$ ) (Figure 4B). In the elderly (age[?]75 years), CRT-D recipients had similar all-cause mortality compared to CRT-P recipients (HR: 0.96, 95\% CI: 0.83-1.12, $\mathrm{I}^{2}=0 \%, \mathrm{P}=0.61$ ) (Figure 4C). Moreover, for patients receiving CRT-D, there was no significant benefits in all-cause mortality compared to CRT-P group regardless of male (HR: $0.83,95 \%$ CI: 0.65-1.07, $\mathrm{I}^{2}=77.8 \%, \mathrm{P}=0.151$ ) (Supplementary Figure 1) and female (HR: 0.87, 95\%CI: $0.54-1.39, \mathrm{I}^{2}=76.5 \%, \mathrm{P}=0.560$ ) (Supplementary Figure 2). Due to high heterogeneity and unstable of results by sensitivity analysis, we cannot draw a conclusion easily in association of gender and device type.

\section{Subgroup analyses}


We performed several subgroup analyses as mentioned below. When restricted the research center to nonEuropean country, we found that CRT-D showed a trend of mortality benefits in all-cause mortality, which did not reach the statistically significant (HR: 0.90, 95\%CI: 0.80-1.01, $\mathrm{I}^{2}=0 \%, \mathrm{P}=0.085$ ) (Supplementary Figure 3). For CRT-D in primary prevention, the pooled HR suggested that additional ICD therapy did not reduce all-cause mortality (HR: 0.92, 95\%CI: 0.74-1.14, $\mathrm{I}^{2}=0 \%, \mathrm{P}=0.444$ ) (Supplementary Figure 4). With long-term follow-up ([?]5 years), CRT-D also did not show advantages in reducing all-cause mortality compared to CRT-P (HR: 0.78, 95\%CI: 0.46-1.34, $\mathrm{I}^{2}=76.4 \%, \mathrm{P}=0.373$ ) (Supplementary Figure 5).

\section{Discussion}

In this meta-analysis, firstly, we have demonstrated that additional ICD therapy shows a mortality benefit in patients undergoing CRT even though using HR adjusted by variables. For patients undergoing CRT-D for primary prevention or from non-European countries, there is no mortality benefit of CRT-D compared with CRT-P. Then, in consideration of the significant difference in clinical characteristic between CRT-P and CRTD recipients, propensity score matching (PSM) was used in several studies to controls the variables more strictly, which eliminated this advantage of CRT-D in all-cause mortality. Finally, in the subpopulation, CRT-D reduced all-cause mortality in ischemic cardiomyopathy patients compared with CRT-P, but this effect is insignificant in non-ischemic cardiomyopathy or older age patients.

\section{CRT-D versus CRT-P}

CRT, as a treatment of HF, has been demonstrated the efficacy and safety in patients with impaired LV function and a wide QRS duration [4,28]. In clinical practice, a high proportion of death among HF patients are due to electrical disturbances, which occur suddenly and unexpectedly. ICD therapy is also recommended in those HF patients for primary prevention to reduce the risk of sudden cardiac death and all-cause mortality [7]. Due to the overlapped indication between two therapies, combined treatment (CRT-D) are going to mainstream gradually. Although no specific recommendation was given to clinicians in choice of CRT-D or CRT-P, the majority patients of US underwent implantation of CRT-D [29]. However, the only randomized trial (COMPANION) did not show a significant difference in mortality between CRT-D and CRT-P directly [4]. A recent meta-analysis, which pooled unadjusted relative risk, revealed an association of additional ICD and lower risk of all-cause mortality in CRT patients [5]. Nevertheless, it is not confident to draw this conclusion unless significant differences in clinical characteristics have been fully considered. Moreover, rate of SCD declined substantially among HF patient with reduced ejection fraction due to increasing use of evidence-based pharmacotherapies [30]. It remains conflicting whether additional ICD can still benefit HF patient receiving CRT.

Due to the absence of strong randomized evidences and recent controversial results, we performed this meta-analysis using adjusted HR to minimize the influence of patient selection bias. In included studies, patients undergoing CRT-P are generally older, less often male gender and less ICM, and these differences are all be corrected by Cox multivariate analysis. Our work indicated a similar result which approve the advantage of additional ICD on CRT patients, but the extent of benefit is decreased compared with previous meta-analysis. Due to the lack of guidance on device selection, clinical practice varies widely around the countries and centers. A cause-of-death analysis of patients with CRT by Marijon et al reported that no statistically difference in SCD incidence between CRT-P and CRT-D, where only $13 \%$ patients implanted CRT-D in secondary prevention. In our subgroup analyses, no benefit was observed when we restricted CRT-D indication to primary prevention [20]. A large observational study by Barra et al suggested a low absolute risk of SCD among CRT patients [11], and Ruwald et al reported a lower risk of ventricular arrhythmias in responders to CRT [31]. Hence, as the cost add-on and inappropriate shock therapy of ICD was brought to consideration [32,33], it may suggest the unnecessity of ICD for CRT patients who did not experience a ventricular arrhythmia causing haemodynamic instability, especially in super-responders. In all included studies with less than 5 years follow-up duration, CRT-D keeps benefits in mortality and there is small amount of heterogeneity across the studies. Limited by few studies reported long-term outcome and nonnegligible heterogeneity, it is still unclear about the performance of CRT-D on long-term ([?]5 years). More studies are needed to illustrate it, which can provide clinical implications to patients with long life 
expectancy. Although CRT-D did not present an advantage in all-cause mortality in non-European countries, we are more inclined to attribute this difference to inconsistence of indication worldwide rather than racial difference [34].

In recent studies, propensity score matching technique has been used in observational studies to adjust for the influence of differences in the clinical characteristics. With more consistent baseline characteristics between CRT-D and CRT-P group, the pooled effect from four included studies did not indicate a lower mortality in patients with CRT-D. However, only part of studies reported HR after propensity matching, and those studies also concluded insignificant results by using adjusted HR. There may be a certain degree of publication bias at all. In addition, another study including 11925 CRT-P recipients and 59534 CRT-D recipients suggested no difference in survival between CRT-P and CRT-D recipients, which used instrumental variable analysis to eliminate the influences of confounders [35]. Different results from different statistic methods offered us an assumption that additional ICD therapy may not benefit CRT patients. As those methods can only eliminate confounders we have known, strong evidence from randomized controlled trials are urgently needed. We are anticipating the result of RESET-CRT clinical trial (ClinicalTrials.gov number NCT03494933), which randomized heart failure patients with LVEF[?]35\% and a CRT indication to either CRT-D or CRT-P implantation.

\section{ICM and NICM}

Supported by strong evidences from RCTs, prophylactic implantation of an ICD, a class I recommendation in HF patient with ICM and reduced left ventricular systolic function, can reduce mortality compared with conventional drug therapy [36,37]. Contrarily, DANISH trials demonstrated that ICD for primary prevention in patients with heart failure caused by non-ischemic etiology did not improve survival benefits [38]. This meta-analysis presented a similar result that ICM patients with CRT indication can benefit from an additional ICD therapy rather than NICM patients. It may be explained by a higher risk of death from fatal arrhythmia in patients with ICM. Moreover, several studies reported that patients with NICM have a better improvement in LV systolic function and LV reverse remodeling compared with patients with ICM $[39,40]$, which resulted in a further reduction of the risk of SCD and ventricular tachyarrhythmias [41]. Above all, it may suggest a less needed of an additional ICD for primary prevention in NICM patients due to a sufficiently reduction of fatal tachyarrhythmias by CRT-P alone.

\section{CRT-D in the Elderly:}

Although current guidelines rarely provide recommendations on the selection of patients for CRT-D and CRT-P, an older age, multiple comorbidity and frail patient is inclined to receive a CRT-P across the centers generally. Our work supported this clinical practice by verifying the non-significant difference of all-cause mortality in the elderly ([?]75 years) between CRT-D and CRT-P. With the progression of HF, pump-failure represents the most frequent cause of death [13,42]. In addition, the risk of death caused by non-cardiac diseases in older patients will increase due to more comorbidities and frailty. Assuming pump-failure and noncardiac death as a competing risk of SCD, the benefits of additional ICD therapy would be attenuated in the elderly. Furthermore, a multicenter observational study by Barra et al including 3008 subjects reported an association of CRT-D and increased occurrence of late complications (a complication occurring or diagnosed following hospital discharge and which had not been seen to occur during the index hospitalization), especially the complication of device-related infection [43]. It may result in a potential increase in admissions and longterm mortality. Meanwhile, Liang et al reported a $5.9 \%$ incidence of inappropriate shock in CRT-D patients, which may affect quality of life and increase the risk of death [33]. As our work indicated that an additional ICD seems to have no advantage in mortality in aged patients, we should not only take clinical index (LVEF, QRS duration) into consideration in aspect of individual device selection, but also patient's age, comorbidities and life expectancy.

\section{Conclusion:}

By pooling the adjusted HR for minimize the influence of clinical differences between CRT-P and CRT-D recipients, our work demonstrates an association of additional ICD and a lower all-cause mortality, especially 
in patients with ICM. CRT-D did not manifest a survival benefits in patients with older age ([?]75 years), NICM, or using for primary prevention, which provides an implication in risk stratification of patients. RCTs are still needed to explore the real effect of additional ICD in mortality.

\section{Limitation:}

The main limitations of this study are as following. Firstly, all the included studies are observational design and significant differences in clinical characteristics between CRT-D and CRT-P were observed. Even though we extracted adjusted HR to minimize the influence of those confounders and made results more reliable, the influence of unknown confounders still cannot be eliminated. Secondly, the majority of including studies are from European; therefore, the effect of additional ICD on all-cause mortality may not be generalizable to population from other areas. Thirdly, sample size varied greatly across the included studies, which means a different statistical power of included studies. However, sensitivity analysis of primary outcome suggested a stable result. Fourthly, limited by high heterogeneity and little data, this work cannot illustrate the benefits of CRT-D in long-term follow-up. Meanwhile, the impact of gender on benefits of CRT-D remains unclear. Finally, patient's quality of life and re-hospitalization rate after implantation are vital clinical index to guide the device selection, but this meta-analysis did not focus on them.

Acknowledgments: Not applicable.

Funding: Not applicable.

Conflicts of interest/Competing interests: Not applicable

Availability of data and material: Not applicable

Code availability: STATA 14 (Stata Corp LP, College Station, Texas)

\section{Reference:}

1. Global, regional, and national incidence, prevalence, and years lived with disability for 301 acute and chronic diseases and injuries in 188 countries, 1990-2013: a systematic analysis for the Global Burden of Disease Study 2013 (2015). Lancet 386 (9995):743-800. doi: 710.1016/S0140-6736(1015)60692-60694.

2. Vaduganathan M, Patel RB, Michel A, Shah SJ, Senni M, Gheorghiade M, et al (2017) Mode of Death in Heart Failure With Preserved Ejection Fraction. J Am Coll Cardiol 69 (5):556-569. doi: $510.1016 /$ j.jacc.2016.1010.1078.

3. van der Meer P, Gaggin HK, Dec GW (2019) ACC/AHA Versus ESC Guidelines on Heart Failure: JACC Guideline Comparison. J Am Coll Cardiol 73 (21):2756-2768. doi: 2710.1016/j.jacc.2019.2703.2478.

4. Bristow MR, Saxon LA, Boehmer J, Krueger S, Kass DA, De Marco T, et al (2004) Cardiacresynchronization therapy with or without an implantable defibrillator in advanced chronic heart failure. N Engl J Med 350 (21):2140-2150. doi: 2110.1056/NEJMoa032423.

5. Barra S, Providência R, Tang A, Heck P, Virdee M, Agarwal S (2015) Importance of Implantable Cardioverter-Defibrillator Back-Up in Cardiac Resynchronization Therapy Recipients: A Systematic Review and Meta-Analysis. J Am Heart Assoc 4 (11):e002539. doi: 002510.001161/JAHA.002115.002539.

6. Döring M, Ebert M, Dagres N, Müssigbrodt A, Bode K, Knopp H, et al (2018) Cardiac resynchronization therapy in the ageing population - With or without an implantable defibrillator? Int J Cardiol 263:48-53. (doi):10.1016/j.ijcard.2018.1003.1087.

7. Ponikowski P, Voors AA, Anker SD, Bueno H, Cleland JGF, Coats AJS, et al (2016) 2016 ESC Guidelines for the diagnosis and treatment of acute and chronic heart failure: The Task Force for the diagnosis and treatment of acute and chronic heart failure of the European Society of Cardiology (ESC)Developed with the special contribution of the Heart Failure Association (HFA) of the ESC. Eur Heart J 37 (27):2129-2200. doi: 2110.1093/eurheartj/ehw2128. 
8. Munir MB, Althouse AD, Rijal S, Shah MB, Abu Daya H, Adelstein E, et al (2016) Clinical Characteristics and Outcomes of Older Cardiac Resynchronization Therapy Recipients Using a Pacemaker versus a Defibrillator. J Cardiovasc Electrophysiol 27 (6):730-734. doi: 710.1111/jce.12951.

9. Witt CT, Kronborg MB, Nohr EA, Mortensen PT, Gerdes C, Jensen HK, et al (2016) Adding the implantable cardioverter-defibrillator to cardiac resynchronization therapy is associated with improved long-term survival in ischaemic, but not in non-ischaemic cardiomyopathy. Europace 18 (3):413-419. doi: 410.1093/europace/euv1212.

10. Barra S, Boveda S, Providência R, Sadoul N, Duehmke R, Reitan C, et al (2017) Adding Defibrillation Therapy to Cardiac Resynchronization on the Basis of the Myocardial Substrate. J Am Coll Cardiol 69 (13):1669-1678. doi: 1610.1016/j.jacc.2017.1601.1042.

11. Barra S, Providência R, Duehmke R, Boveda S, Marijon E, Reitan C, et al (2017) Sex-specific outcomes with addition of defibrillation to resynchronisation therapy in patients with heart failure. Heart 103 (10):753760. doi: 710.1136/heartjnl-2016-310677.

12. Laish-Farkash A, Bruoha S, Katz A, Goldenberg I, Suleiman M, Michowitz Y, et al (2017) Morbidity and mortality with cardiac resynchronization therapy with pacing vs. with defibrillation in octogenarian patients in a real-world setting. Europace 19 (8):1357-1363. doi: 1310.1093/europace/euw1238.

13. Martens P, Verbrugge FH, Nijst P, Dupont M, Nuyens D, Herendael HV, et al (2017) Incremental benefit of cardiac resynchronisation therapy with versus without a defibrillator. Heart 103 (24):1977-1984. doi: 1910.1136/heartjnl-2017-311423.

14. Yokoshiki H, Shimizu A, Mitsuhashi T, Furushima H, Sekiguchi Y, Manaka T, et al (2017) Survival and Heart Failure Hospitalization in Patients With Cardiac Resynchronization Therapy With or Without a Defibrillator for Primary Prevention in Japan - Analysis of the Japan Cardiac Device Treatment Registry Database. Circ J 81 (12):1798-1806. doi: 1710.1253/circj.CJ-1717-0234.

15. Leyva F, Qiu T, Zegard A, McNulty D, Evison F, Ray D, et al (2019) Sex-Specific Differences in Survival and Heart Failure Hospitalization After Cardiac Resynchronization Therapy With or Without Defibrillation. J Am Heart Assoc 8 (22):e013485. doi: 013410.011161/JAHA.013119.013485.

16. Leyva F, Zegard A, Okafor O, de Bono J, McNulty D, Ahmed A, et al (2019) Survival after cardiac resynchronization therapy: results from 50084 implantations. Europace 21 (5):754-762. doi: 710.1093/europace/euy1267.

17. Saba S, McLaughlin T, He M, Althouse A, Mulukutla S, Hernandez I (2019) Cardiac resynchronization therapy using pacemakers vs defibrillators in patients with nonischemic cardiomyopathy: The United States experience from 2007 to 2014. Heart Rhythm 16 (7):1065-1071. doi: 1010.1016/j.hrthm.2019.1004.1028.

18. Wang Y, Sharbaugh MS, Althouse AD, Mulukutla S, Saba S (2019) Cardiac resynchronization therapy pacemakers versus defibrillators in older non-ischemic cardiomyopathy patients. Indian Pacing Electrophysiol J 19 (1):4-6. doi: 10.1016/j.ipej.2018.1008.1002.

19. Liang Y, Wang J, Yu Z, Zhang M, Pan L, Nie Y, et al (2020) Comparison between cardiac resynchronization therapy with and without defibrillator on long-term mortality: A propensity score matched analysis. J Cardiol 75 (4):432-438. doi: 410.1016/j.jjcc.2019.1008.1018.

20. Marijon E, Leclercq C, Narayanan K, Boveda S, Klug D, Lacaze-Gadonneix J, et al (2015) Causes-ofdeath analysis of patients with cardiac resynchronization therapy: An analysis of the CeRtiTuDe cohort study. European Heart Journal 36 (41):2767-2776. doi: 10.1093/eurheartj/ehv455

21. Pappone C, Vicedomini G, Augello G, Mazzone P, Nardi S, Rosanio S (2003) Combining electrical therapies for advanced heart failure: the Milan experience with biventricular pacing-defibrillation backup combination for primary prevention of sudden cardiac death. Am J Cardiol 91 (9A):74F-80F. doi: 10.1016/s00029149(1002)03341-03346. 
22. Kutyifa V, Geller L, Bogyi P, Zima E, Aktas MK, Ozcan EE, et al (2014) Effect of cardiac resynchronization therapy with implantable cardioverter defibrillator versus cardiac resynchronization therapy with pacemaker on mortality in heart failure patients: results of a high-volume, single-centre experience. Eur J Heart Fail 16 (12):1323-1330. doi: 1310.1002/ejhf.1185.

23. Gillebert C, Marynissen T, Janssen R, Droogne W, Voros G, Garweg C, et al (2014) How to choose between a pacemaker or defibrillator for resynchronization therapy? Acta Cardiol 69 (5):483-489. doi: 410.1080/ac.1069.1085.3044874.

24. Gold MR, Daubert JC, Abraham WT, Hassager C, Dinerman JL, Hudnall JH, et al (2013) Implantable defibrillators improve survival in patients with mildly symptomatic heart failure receiving cardiac resynchronization therapy: analysis of the long-term follow-up of remodeling in systolic left ventricular dysfunction (REVERSE). Circ Arrhythm Electrophysiol 6 (6):1163-1168. doi: 1110.1161/CIRCEP.1113.000570.

25. Reitan C, Chaudhry U, Bakos Z, Brandt J, Wang L, Platonov PG, et al (2015) Long-term results of cardiac resynchronization therapy: A comparison between crt-pacemakers versus primary prophylactic CRT-defibrillators. PACE - Pacing and Clinical Electrophysiology 38 (6):758-767. doi:10.1111/pace.12631

26. Killu AM, Wu JH, Friedman PA, Shen WK, Webster TL, Brooke KL, et al (2013) Outcomes of cardiac resynchronization therapy in the elderly. Pacing Clin Electrophysiol 36 (6):664-672. doi: $610.1111 /$ pace. 12048 .

27. Morani G, Gasparini M, Zanon F, Casali E, Spotti A, Reggiani A, et al (2013) Cardiac resynchronization therapy-defibrillator improves long-term survival compared with cardiac resynchronization therapypacemaker in patients with a class IA indication for cardiac resynchronization therapy: data from the Contak Italian Registry. Europace 15 (9):1273-1279. doi: 1210.1093/europace/eut1032.

28. Cleland JG, Daubert JC, Erdmann E, Freemantle N, Gras D, Kappenberger L, et al (2005) The effect of cardiac resynchronization on morbidity and mortality in heart failure. N Engl J Med 352 (15):1539-1549. doi: 1510.1056/NEJMoa050496.

29. Lindvall C, Chatterjee NA, Chang Y, Chernack B, Jackson VA, Singh JP, et al (2016) National Trends in the Use of Cardiac Resynchronization Therapy With or Without Implantable Cardioverter-Defibrillator. Circulation 133 (3):273-281. doi: 210.1161/CIRCULATIONAHA.1115.018830.

30. Shen L, Jhund PS, Petrie MC, Claggett BL, Barlera S, Cleland JGF, et al (2017) Declining Risk of Sudden Death in Heart Failure. N Engl J Med 377 (1):41-51. doi: 10.1056/NEJMoa1609758.

31. Ruwald MH, Solomon SD, Foster E, Kutyifa V, Ruwald AC, Sherazi S, et al (2014) Left ventricular ejection fraction normalization in cardiac resynchronization therapy and risk of ventricular arrhythmias and clinical outcomes: results from the Multicenter Automatic Defibrillator Implantation Trial With Cardiac Resynchronization Therapy (MADIT-CRT) trial. Circulation 130 (25):2278-2286. doi: 2210.1161/CIRCULATIONAHA.2114.011283.

32. Daubert JP, Zareba W, Cannom DS, McNitt S, Rosero SZ, Wang P, et al (2008) Inappropriate implantable cardioverter-defibrillator shocks in MADIT II: frequency, mechanisms, predictors, and survival impact. J Am Coll Cardiol 51 (14):1357-1365. doi: 1310.1016/j.jacc.2007.1309.1073.

33. Poole JE, Johnson GW, Hellkamp AS, Anderson J, Callans DJ, Raitt MH, et al (2008) Prognostic importance of defibrillator shocks in patients with heart failure. N Engl J Med 359 (10):1009-1017. doi: 1010.1056/NEJMoa071098.

34. Ziaeian B, Zhang Y, Albert NM, Curtis AB, Gheorghiade M, Heywood JT, et al (2014) Clinical effectiveness of CRT and ICD therapy in heart failure patients by racial/ethnic classification: insights from the IMPROVE HF registry. J Am Coll Cardiol 64 (8):797-807. doi: 710.1016/j.jacc.2014.1005.1060.

35. Kramer DB, Normand ST, Volya R, Hatfield LA (2018) Facility-Level Variation and Clinical Outcomes in Use of Cardiac Resynchronization Therapy With and Without an Implantable Cardioverter-Defibrillator. 
Circ Cardiovasc Qual Outcomes 11 (12):e004763. doi: 004710.001161/CIRCOUTCOMES.004118.004763.

36. Improved survival with an implanted defibrillator in patients with coronary disease at high risk for ventricular arrhythmia (1997). Circulation 95 (4):777. doi: 710.1161/1101.cir.1195.1164.1777.

37. Buxton AE, Lee KL, Fisher JD, Josephson ME, Prystowsky EN, Hafley G (1999) A randomized study of the prevention of sudden death in patients with coronary artery disease. Multicenter Unsustained Tachycardia Trial Investigators. N Engl J Med 341 (25):1882-1890. doi: 1810.1056/NEJM199912163412503.

38. Køber L, Thune JJ, Nielsen JC, Haarbo J, Videbæk L, Korup E, et al (2016) Defibrillator Implantation in Patients with Nonischemic Systolic Heart Failure. N Engl J Med 375 (13):1221-1230. doi: 1210.1056/NEJMoa1608029.

39. Duncan A, Wait D, Gibson D, Daubert JC (2003) Left ventricular remodelling and haemodynamic effects of multisite biventricular pacing in patients with left ventricular systolic dysfunction and activation disturbances in sinus rhythm: sub-study of the MUSTIC (Multisite Stimulationin Cardiomyopathies) trial. Eur Heart J 24 (5):430-441. doi: 410.1016/s0195-1668x(1002)00475-x.

40. Sutton MG, Plappert T, Hilpisch KE, Abraham WT, Hayes DL, Chinchoy E (2006) Sustained reverse left ventricular structural remodeling with cardiac resynchronization at one year is a function of etiology: quantitative Doppler echocardiographic evidence from the Multicenter InSync Randomized Clinical Evaluation (MIRACLE). Circulation 113 (2):266-272. doi: 210.1161/CIRCULATIONAHA.1104.520817.

41. Barsheshet A, Wang PJ, Moss AJ, Solomon SD, Al-Ahmad A, McNitt S, et al (2011) Reverse remodeling and the risk of ventricular tachyarrhythmias in the MADIT-CRT (Multicenter Automatic Defibrillator Implantation Trial-Cardiac Resynchronization Therapy). J Am Coll Cardiol 57 (24):2416-2423. doi: $2410.1016 /$ j.jacc.2010.2412.2041.

42. Barra S, Providência R, Duehmke R, Boveda S, Begley D, Grace A, et al (2018) Cause-of-death analysis in patients with cardiac resynchronization therapy with or without a defibrillator: a systematic review and proportional meta-analysis. Europace 20 (3):481-491. doi: 410.1093/europace/eux1094.

43. Barra S, Providência R, Boveda S, Duehmke R, Narayanan K, Chow AW, et al (2018) Device complications with addition of defibrillation to cardiac resynchronisation therapy for primary prevention. Heart 104 (18):1529-1535. doi: 1510.1136/heartjnl-2017-312546.

\section{Hosted file}

Table 1.docx available at https://authorea.com/users/329109/articles/456174-the-benefitsof-additional-defibrillator-in-heart-failure-patients-with-cardiac-resynchronizationtherapy-a-meta-analysis-of-observational-studies 


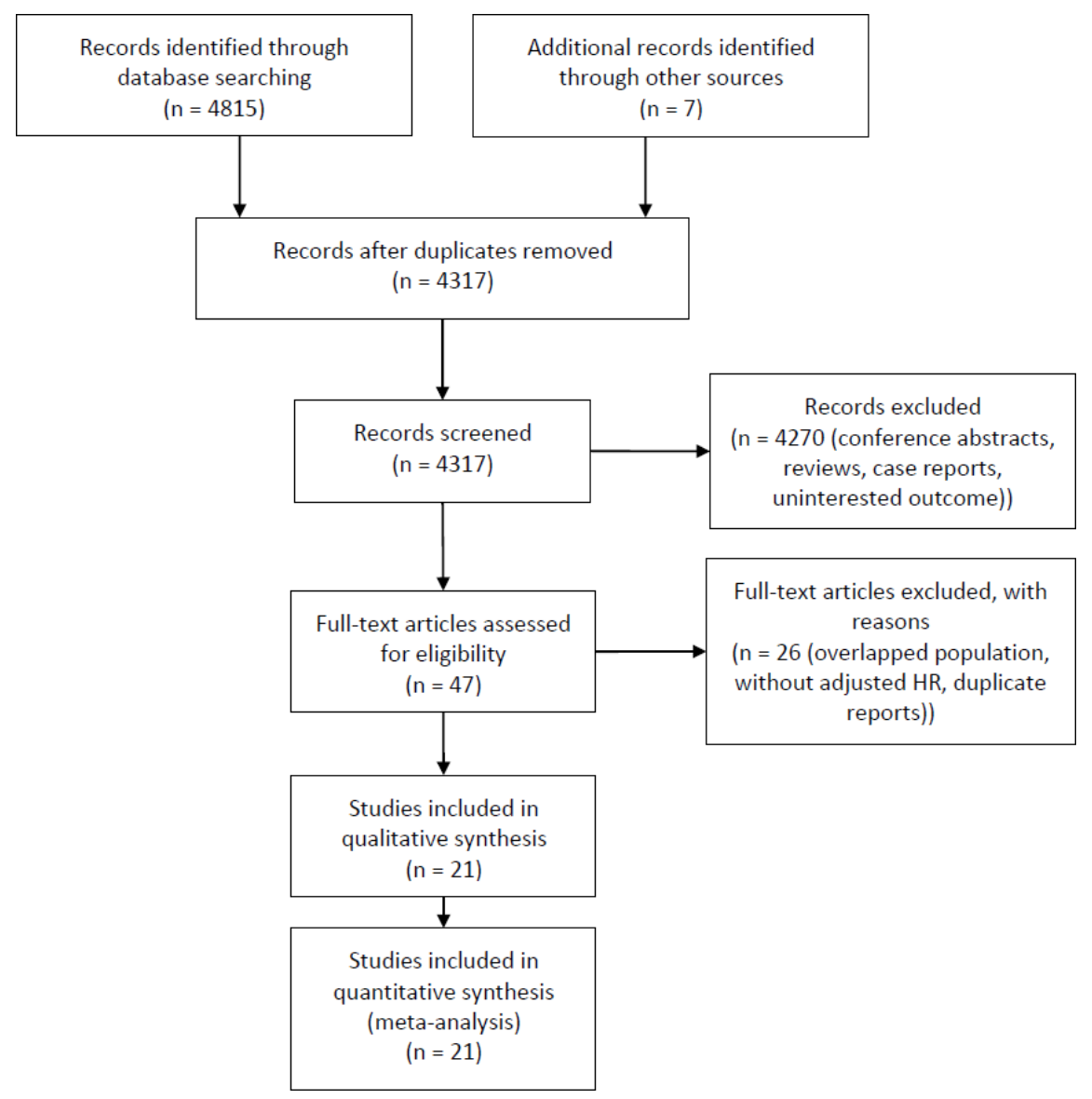



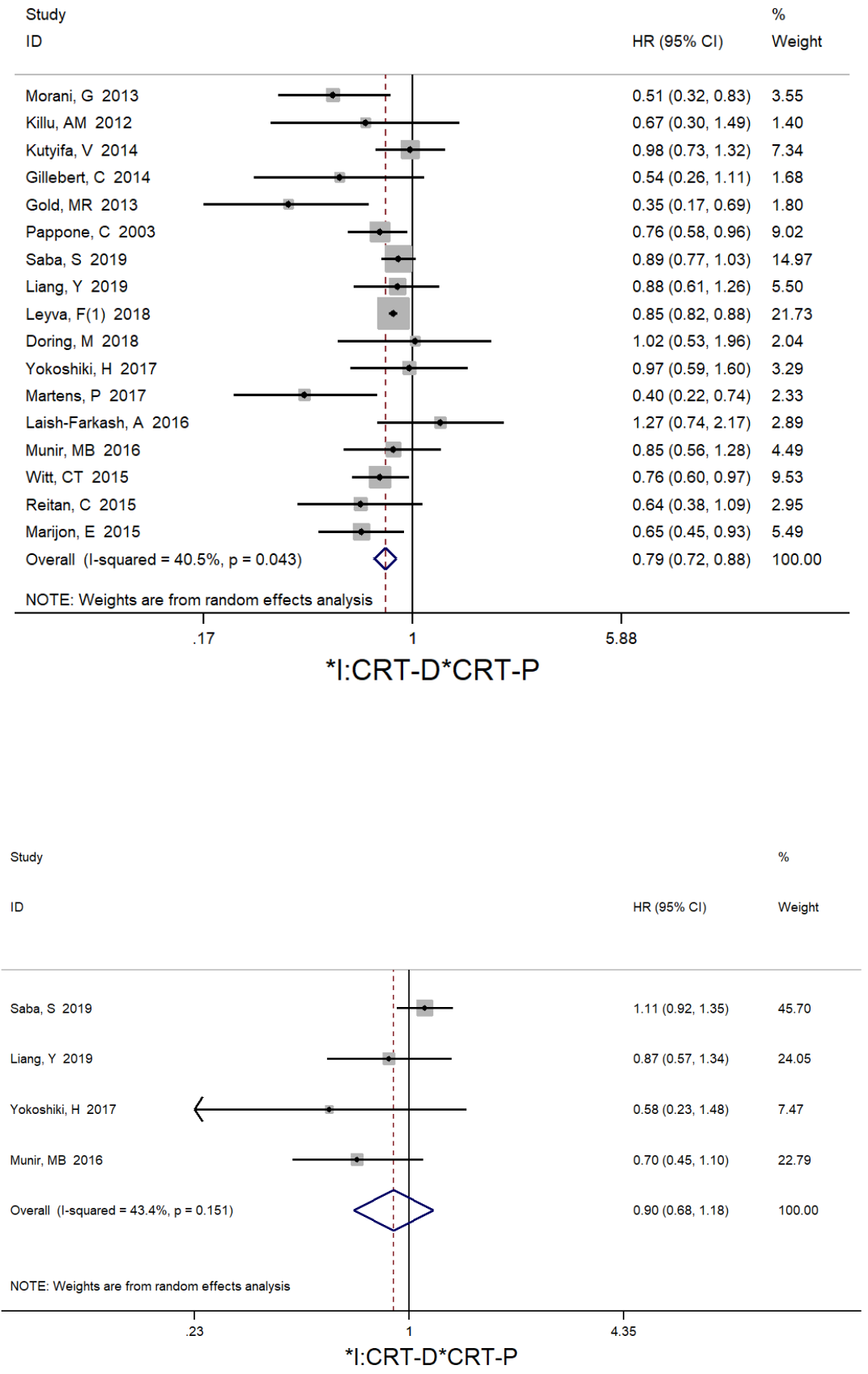


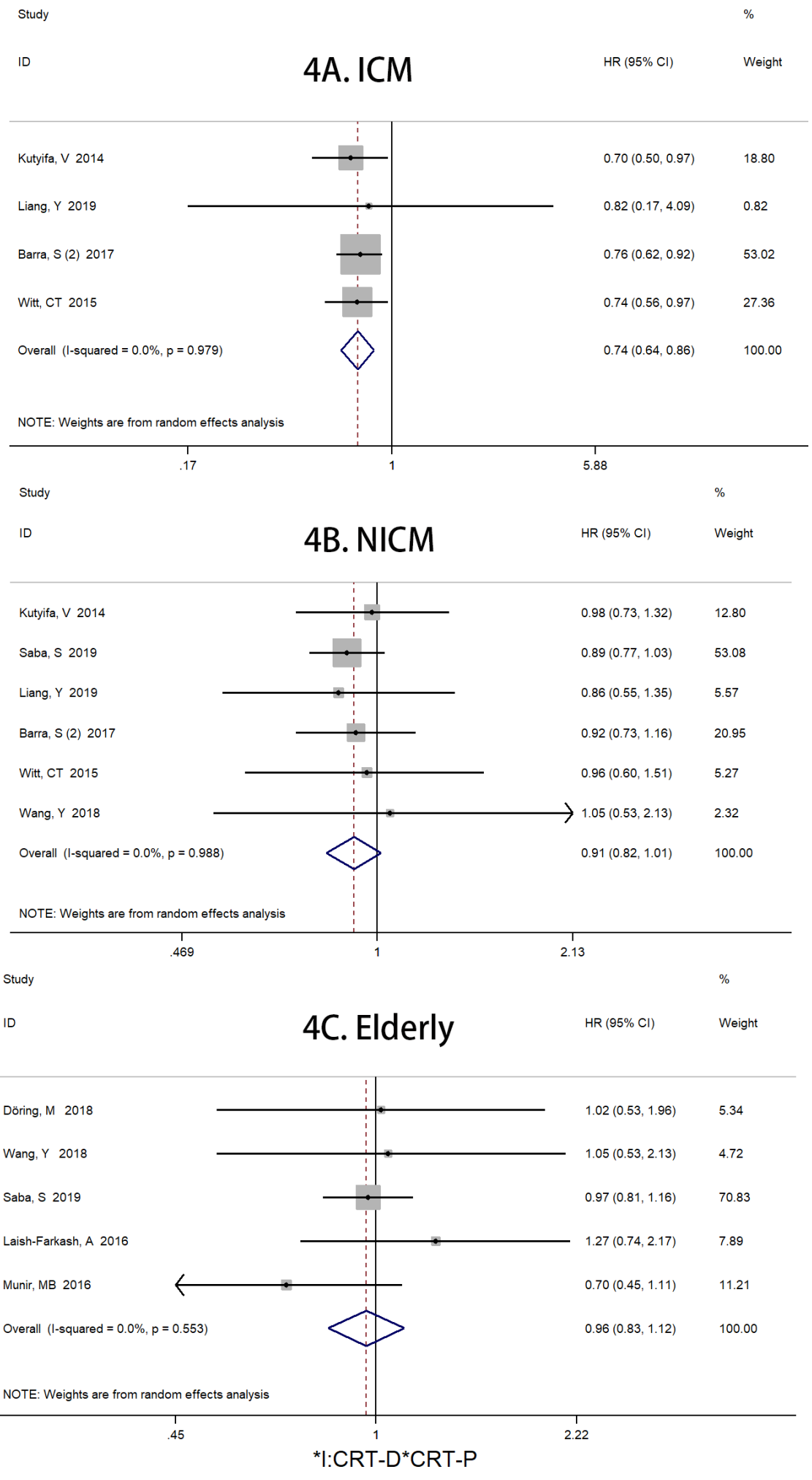

